\title{
Complex magnetic behavior and high spin polarization in $\mathrm{Fe}_{3-x} \mathrm{Mn}_{x} \mathrm{Si}$ alloys
}

\author{
Marjana Ležaić, ${ }^{*}$ Phivos Mavropoulos, ${ }^{\dagger}$ and Stefan Blügel \\ Peter Grünberg Institut and Institute for Advanced Simulation, Forschungszentrum Jülich and JARA, D-52425 Jülich, Germany
}

Hubert Ebert

Department Chemie/Physikalische Chemie, Ludwig-Maximillians-Universität München, Butenandtstrasse 5-13, D-81377 München, Germany

(Received 16 December 2010; published 30 March 2011)

\begin{abstract}
$\mathrm{Fe}_{3} \mathrm{Si}$ is a ferromagnetic material with possible applications in magnetic tunnel junctions. When doped with $\mathrm{Mn}$, the material shows a complex magnetic behavior, as suggested by older experiments. We employed the Korringa-Kohn-Rostoker Green-function method within density-functional theory in order to study the alloy $\mathrm{Fe}_{3-x} \mathrm{Mn}_{x} \mathrm{Si}$, with $0 \leqslant x \leqslant 1$. Chemical disorder is described within the coherent potential approximation. In agreement with experiment, we find that the Mn atoms align ferromagnetically to the Fe atoms, and that the magnetization and Curie temperature drop with increasing Mn concentration $x$. The calculated spin polarization $P$ at the Fermi level varies strongly with $x$, from $P=-0.3$ at $x=0$ (ordered $\mathrm{Fe}_{3} \mathrm{Si}$ ) through $P=0$ at $x=0.28$, to $P=+1$ for $x>0.75$; i.e., at high Mn concentrations the system is half metallic. We discuss the origin of the trends of magnetic moments, exchange interactions, Curie temperature, and the spin polarization.
\end{abstract}

DOI: 10.1103/PhysRevB.83.094434

PACS number(s): 75.50.Bb, 71.20.Be, 71.70.Gm, 71.20.Lp

\section{INTRODUCTION}

Magnetic intermetallic alloys show very rich physics depending on the degree of doping and chemical disorder, which can therefore be used as "control parameters" allowing us to tune the electronic and magnetic structure for desired effects. In particular, physical properties that are fundamental for technological applications in spintronics, such as the magnetization $M$, Curie temperature $T_{\mathrm{C}}$, or spin polarization $P$ at the Fermi energy, vary strongly with respect to these control parameters.

The alloy $\mathrm{Fe}_{3-x} \mathrm{Mn}_{x} \mathrm{Si}$, belonging to the wider class of $\mathrm{Fe}_{3-x} \mathrm{TM}_{x}$ Si alloys with TM a transition-metal element, ${ }^{1}$ is an example of such dependence on the degree of doping. ${ }^{2}$ As the $\mathrm{Mn}$ concentration increases, the magnetization of $\mathrm{Fe}_{3-x} \mathrm{Mn}_{x} \mathrm{Si}$ drops continuously from $5 \mu_{\mathrm{B}}$ to zero; its temperaturedependent magnetic properties change from high- $T_{\mathrm{C}}$ $(\approx 800 \mathrm{~K})$ ferromagnetism, through low $-T_{\mathrm{C}}$ ferromagnetism with re-entrant behavior at $70 \mathrm{~K}$, to complex noncollinear magnetism; its calculated spin polarization increases from $-30 \%$ to the ideal, half metallic $+100 \%,{ }^{3}$ and then drops again due to the noncollinear behavior. These observations are not new, however, there is a recent revival of the interest in $\mathrm{Fe}_{3-x} \mathrm{Mn}_{x} \mathrm{Si}$ due to potential applications in magnetic tunnel junctions. $^{4}$

Motivated by this revival, we present here a theoretical study of the electronic and magnetic properties of $\mathrm{Fe}_{3-x} \mathrm{Mn}_{x} \mathrm{Si}$ for $0<x<1$ based on ab initio calculations. The choice of concentration range is motivated by the specific site preference of $\mathrm{Mn}$ for $x<1$, so that the resulting state is ferromagnetic as we see later; for $x>1$, noncollinear magnetic configurations can occur. We provide an interpretation of the magnetization drop as a function of concentration in terms of wave-function symmetry and hybridization, together with the requirement for local charge neutrality. We further propose that the increase in spin polarization up to the half metallic point is due to the same mechanisms that cause the magnetization drop. Moreover, after extracting exchange interactions from the $a b$ initio results, we calculate the Curie temperature using a
Monte Carlo approach, and are able to reproduce the drop of $T_{\mathrm{C}}$ as a function of $\mathrm{Mn}$ concentration. Finally, we discuss where our results do not agree with experiment, and we propose a possible reason for the disagreement; this is particularly the case for the re-entrant behavior and the value of magnetization at high Mn concentrations.

The paper is structured as follows. In Sec. II we summarize the experimental and theoretical background on $\mathrm{Fe}_{3-x} \mathrm{Mn}_{x} \mathrm{Si}$. Section III is devoted to the description of our calculational approach. We continue with a presentation of our results on the magnetization and spin polarization in Secs. IV and V and of the Curie temperature in Sec. VI. In Sec. VII we discuss the limitations of our approach, their consequences, and possibilities for a more accurate description. Our conclusions are summarized in Sec. VIII.

\section{EXPERIMENTAL AND THEORETICAL BACKGROUND}

\section{A. Experiment}

Quite a few experimental studies have been done on the magnetic properties of $\mathrm{Fe}_{3-x} \mathrm{Mn}_{x} \mathrm{Si}$, revealing a highly complex magnetic behavior dependent on the Mn concentration $x$. Here we recall the main results of these experiments, with emphasis on the concentration range $0<x<1$, which interests us in the present work. At high concentrations, one reaches the $\mathrm{Fe}$-doped $\mathrm{Mn}_{3} \mathrm{Si}$ compound, which exhibits more complicated properties; e.g., $\mathrm{Mn}_{3} \mathrm{Si}$ is an incommensurate antiferromagnet with a Néel temperature of about $25 \mathrm{~K}$, while $\mathrm{Fe}_{3} \mathrm{Si}$ is a ferromagnet.

Structure and site preference. $\mathrm{Fe}_{3} \mathrm{Si}$ crystallizes in the $\mathrm{DO}_{3}$ structure consisting of a fcc lattice with four basis atoms (see Fig. 1). These are placed at $(0,0,0) a$ (Si atom), $\left(\frac{1}{4}, \frac{1}{4}, \frac{1}{4}\right) a$ $\left(\mathrm{Fe}_{\mathrm{A}}\right.$ atom), $\left(\frac{1}{2}, \frac{1}{2}, \frac{1}{2}\right) a$ ( $\mathrm{Fe}_{\mathrm{B}}$ atom), $\left(\frac{3}{4}, \frac{3}{4}, \frac{3}{4}\right) a$ ( $\mathrm{Fe}_{\mathrm{C}}$ atom), where $a$ is the lattice constant. The $\mathrm{Fe}_{\mathrm{A}}$ and $\mathrm{Fe}_{\mathrm{C}}$ atoms are tetrahedrally coordinated to four $\mathrm{Fe}_{\mathrm{B}}$ and four $\mathrm{Si}$ atoms and exhibit equivalent electronic ground-state properties due to symmetry. The $\mathrm{Fe}_{\mathrm{B}}$ atoms are octahedrally coordinated to eight 

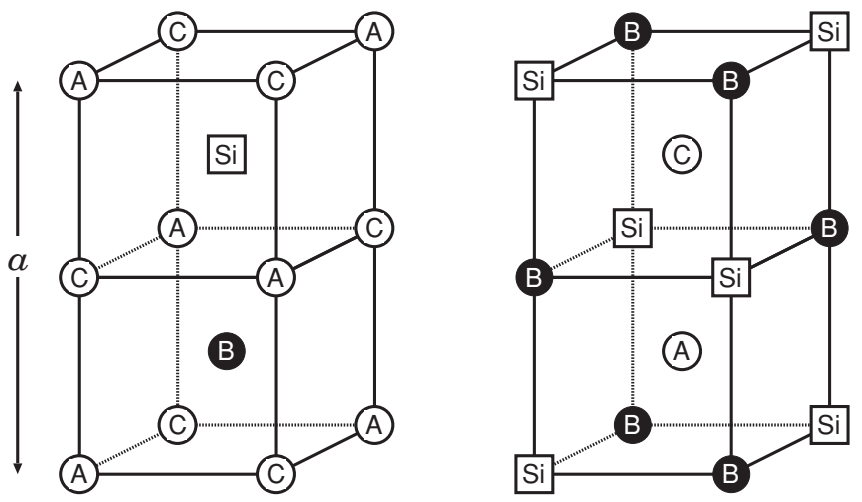

FIG. 1. Geometrical structure of $\mathrm{Fe}_{3} \mathrm{Si}$ and $\mathrm{Fe}_{3-x} \mathrm{Mn}_{x} \mathrm{Si}$. The $A$ and $C$ sites are occupied by $\mathrm{Fe}$, while the $B$ site is occupied either by $\mathrm{Fe}$ or by Mn. Square symbols show the positions of the $\mathrm{Si}$ atoms. In the left panel we show the octahedral coordination of the $B$ site to $A$ and $C$ Fe neighbors, while in the right panel we show the tetrahedral environment of the $A$ and $C$ sites.

$\mathrm{Fe}_{\mathrm{A}, \mathrm{C}}$ atoms; their electronic properties therefore resemble somewhat bcc Fe, as we will discuss.

When $\mathrm{Mn}$ is doped into $\mathrm{Fe}_{3} \mathrm{Si}$, it substitutes $\mathrm{Fe}_{\mathrm{B}}$ atoms, as is found by experiment. This appears to be part of a general trend found experimentally ${ }^{1,5,6}$ and modeled theoretically ${ }^{7}$ in which transition-element atoms which are to the left of Fe in the periodic table prefer to reside at the $B$ site when doped into $\mathrm{Fe}_{3} \mathrm{Si}$, while transition-element atoms which are to the right of Fe prefer to substitute the Fe atoms at the $A$ and $C$ sites.

Yoon and Booth ${ }^{2,8}$ report that, in the range $0<x<0.75$, the $\mathrm{Mn}$ atoms substitute the $B$-site $\mathrm{Fe}$, as verified by hyperfinefield measurements of Niculescu et al. ${ }^{1,9}$ Above $x=0.75$, an increasing fraction of $\mathrm{Mn}$ atoms tends to reside at the $A, C$ sites with equal probability. At $x=1$, this "swap" fraction is $12 \%$ according to Ziebeck and Webster ${ }^{10}$ and $15 \%$ according to Yoon and Booth ${ }^{2}$ i.e., even $\mathrm{Fe}_{2} \mathrm{MnSi}$ contains a small amount of chemical disorder. $\mathrm{Fe}_{3-x} \mathrm{Mn}_{x} \mathrm{Si}$ has thus the $L 2_{1}$, or fullHeusler, structure. Similar results are found in more recent hyperfine-field experiments. ${ }^{11}$

The measured lattice parameter changes linearly and only slightly as a function of the concentration $x$ (see Ref. 9) from $5.653 \AA$ at $x=0$ to $5.663 \AA$ at $x=1$, i.e., by about $0.2 \%$. The linear change continues for higher $x$.

Magnetic moments. As was shown by Booth et al. ${ }^{12}$ and Yoon and Booth, ${ }^{2,8}$ the saturation magnetization $M$ drops linearly from about $4.8 \mu_{\mathrm{B}}$ to $2.6 \mu_{\mathrm{B}}$ per formula unit in the range $0<x<0.75$. The local magnetic moments depend strongly on the site. The $B$ site has a high moment of about $2.3 \pm 0.3 \mu_{\mathrm{B}}$ in the range $0 \leqslant x<0.75$, which then drops gradually and vanishes at $x=1.75$; at the $A, C$ sites (containing only $\mathrm{Fe}$ for low $x$ ), the moment decreases from $1.4 \mu_{\mathrm{B}}$ to $0.3 \mu_{\mathrm{B}}$ as $x$ increases from 0 to 0.75 . The net result is the aforementioned drop of the total magnetic moment.

Anomalous temperature dependence of the magnetization. In the concentration range $0.75<x<1.75$ a re-entrant behavior of the magnetization curve $M(T)$ is found:2,8 For temperatures from $T=0 \mathrm{~K}$ up to the re-entry temperature $T_{\mathrm{R}} \approx 70 \mathrm{~K}, M(T)$ is increasing, while for $T>T_{\mathrm{R}} M(T)$ is decreasing, as expected for a usual ferromagnet, up to the Curie temperature $T_{\mathrm{C}}$. Thus two values of the saturation magnetization can be defined, one $\left(M_{0}\right)$ as the actual measured value $M(T=0)$, and another $\left(M_{\text {extr }}\right)$ as the extrapolated value of $M(T)$ from data taken for $T>T_{\mathrm{R}}$; evidently, $M_{\text {extr }}>M_{0}$. For $x=0.75$, in fact, where this effect just starts to appear, the two values are close $\left(M_{0} \approx 0.95 M_{\text {extr }}\right)$, and application of a magnetic field of $14 \mathrm{kOe}$ can further saturate the sample so as to reach the value $M_{\text {extr }}$. The anomalous temperature dependence of $M$ was also found by Ziebeck and Webster ${ }^{10}$ for the $\mathrm{Fe}_{2} \mathrm{MnSi}$ alloy, as well as by Nagano and co-workers ${ }^{13}$ and by Ersez et al. ${ }^{14}$ who analyzed the effect via neutron scattering. However, in a recent work where the samples were grown by molecular beam epitaxy, ${ }^{4}$ this anomalous behavior was not observed (up to $x=1.4$ ).

Curie temperature. The Curie temperature drops as a function of the Mn concentration $x$, from about $T_{\mathrm{C}} \approx 800 \mathrm{~K}$ at $x=0$ to $T_{\mathrm{C}}=0 \mathrm{~K}$ at $x \approx 1.75$; i.e., for $x>1.75$ no saturation magnetization is found, while the sample is at a complex noncollinear magnetic state. At $x=0.75$, where the re-entrant magnetic behavior sets in, $T_{\mathrm{C}}=375 \mathrm{~K}$ and $T_{\mathrm{R}} \approx 40 \mathrm{~K}$, while at $x=1, T_{\mathrm{C}} \approx 220 \mathrm{~K}$ and $T_{\mathrm{R}} \approx 70 \mathrm{~K} .^{2}$

Thermodynamic properties. The anomalous behavior at $T_{\mathrm{R}}$ also shows in measurements of thermodynamic quantities. In particular, Smith et al. ${ }^{15}$ have measured a specific-heat anomaly at $T_{\mathrm{R}}$, most pronounced for the $x=1$ compounds. Furthermore, Miles et al. ${ }^{16}$ report a sharp peak of the thermalexpansion coefficient at $T_{\mathrm{R}}=60 \mathrm{~K}$ for the $\mathrm{Fe}_{2} \mathrm{MnSi}$ alloy (i.e., $x=1$ ), while they find no such pronounced behavior at $T_{\mathrm{C}}$.

Heat treatment. As reported in Ref. 2, the sample preparation included a $24-\mathrm{h}$ heating at $830^{\circ} \mathrm{C}$ and water quenching; a different heat treatment ( 21 days at $550{ }^{\circ} \mathrm{C}$ and slow cooling) for samples with $0.95<x<1.25$ appeared to increase $M_{0}$, bringing it closer to $M_{\text {extr }}$, but had no effect on the values of $T_{\mathrm{R}}$ or $T_{\mathrm{C}}$. Ziebeck and Webster ${ }^{10}$ and Smith et al. ${ }^{15}$ also used an annealing treatment at over $800^{\circ} \mathrm{C}$ for $24 \mathrm{~h}$ and water quenching. Miles et al. ${ }^{16}$ used two samples, one quenched from $800{ }^{\circ} \mathrm{C}$ and one slowly cooled, with no change in the expansion coefficient and its anomaly at $T_{\mathrm{R}}$.

\section{B. Theory}

Only few theoretical results exist on the electronic structure of $\mathrm{Fe}_{3-x} \mathrm{Mn}_{x} \mathrm{Si}$. Mohn and Supanetz ${ }^{17}$ employed an augmented spherical wave method and the local spin-density approximation (LSDA) to density-functional theory to examine noncollinear states in the ordered alloys $\mathrm{Fe}_{3} \mathrm{Si}, \mathrm{Fe}_{2} \mathrm{MnSi}$ (with the $\mathrm{Mn}$ atom at the $B$ site), $\mathrm{FeMn}{ }_{2} \mathrm{Si}$ (with one $\mathrm{Mn}$ atom at the $B$ site and one at the $A$ site), and $\mathrm{Mn}_{3} \mathrm{Si}$. For $\mathrm{Fe}_{3} \mathrm{Si}$ they found a ferromagnetic ground state, while noncollinear ground states were found for all other compounds. In particular for $\mathrm{Fe}_{2} \mathrm{MnSi}$, which is of interest here, Mohn and Supanetz ${ }^{17}$ found a local energy minimum at the ferromagnetic state, with a lower minimum for a spin spiral along the [111] axis, at a q vector of $\frac{2 \pi}{a}(0.45,0.45,0.45)$. According to their calculation, the ground state was an antiferromagnetic state, with the Mn moments alternating along the [111] axis, while the moment direction was canted off the [111] direction by about $60^{\circ}$. The energy difference between the noncollinear ground state and the ferromagnetic state in $\mathrm{Fe}_{2} \mathrm{MnSi}$ was reported to be around $0.8 \mathrm{mRyd}(10.9 \mathrm{meV})$. A canting of the magnetic 
moments below $T_{\mathrm{R}}$ was also assumed by Yoon and Booth ${ }^{2,8}$ in order to explain the neutron-scattering data.

More recently, ab initio calculations were presented ${ }^{18}$ on $\mathrm{Fe}_{3-x} \mathrm{Mn}_{x} \mathrm{Si}$ for $0 \leqslant x \leqslant 0.5$ using the supercell method. Mn was considered to occupy the $B$ site. It was found that the spin moments of $\mathrm{Fe}$ at the $A$ and $C$ sites are reduced in the presence of Mn nearest neighbors, which induces a redistribution of the $\mathrm{Fe}_{\mathrm{A}, \mathrm{C}}$ states; a drop of the total moment with increasing Mn concentration was observed and attributed to the $\mathrm{Fe}_{\mathrm{A}, \mathrm{C}}$ moments. Furthermore, ab initio calculations on $\mathrm{Fe}_{3-x} \mathrm{Mn}_{x} \mathrm{Si}$ and $\mathrm{Fe}_{3-x} \mathrm{MnSi}_{x}$ alloys were presented in Ref. 19; the calculations here agree with the previous results that the magnetic moments of the $A$ and $C$ sites drop as a function of Mn concentration, while it is found that the $B$-site atomic moments increase. The Fe moments appear to be higher than the Mn moments, so that the $B$-site average moment does not change much.

Further theoretical work appears in parallel with experiments. Szymański et al. ${ }^{20}$ examined the spin dynamics of $\mathrm{Fe}_{3-x} \mathrm{Mn}_{x} \mathrm{Si}$ using neutron scattering at room temperature and at liquid-nitrogen temperature, and fitted their results to effective interatomic exchange integrals which enter a Heisenberg Hamiltonian. The fitted values of the exchange constants depend on the number of neighbors considered; the nearest-neighbor exchange for $\mathrm{Fe}_{3} \mathrm{Si}$ ranges between 10 and $20 \mathrm{meV}$. Brown and co-workers ${ }^{21}$ analyze the behavior of the magnetic moments based on symmetry arguments and on a model by Swintendick ${ }^{7}$ and conclude that, as the Mn concentration increases, the reduction of the exchange splitting leads to the drop of the $\mathrm{Fe}_{\mathrm{A}, \mathrm{C}}$ moment.

\section{METHOD OF CALCULATION, MODELS, AND CUTOFF PARAMETERS}

Our electronic-structure calculations are based on densityfunctional theory within the generalized gradient approximation $(\mathrm{GGA})^{22}$ to account for exchange and correlation effects. The local spin-density approximation ${ }^{23}$ was also used for comparison and proved to be inadequate for the prediction of the correct magnetic ground state at low and intermediate Mn concentrations (see Sec. IV). Calculations were also performed within the "single-shot GGA," ${ }^{24}$ where, using the self-consistent LSDA spin density, $\rho_{\mathrm{LSDA}}$, the total energy is calculated within the GGA functional $E_{\mathrm{GGA}}[\rho]$; i.e., one calculates $E_{\mathrm{GGA}}\left[\rho_{\mathrm{LSDA}}\right]$. This approach is based on the idea that $\rho_{\text {LSDA }}$, as a trial density, is not too far from the self-consistent GGA density $\rho_{\mathrm{GGA}}$, so that, due to the variational character of the energy functional, $E_{\mathrm{GGA}}\left[\rho_{\mathrm{LSDA}}\right] \approx E_{\mathrm{GGA}}\left[\rho_{\mathrm{GGA}}\right]$. The single-shot GGA is known, for instance, to correct the LSDA overbinding, giving an improved equilibrium lattice parameter, very close to the one predicted by the GGA. ${ }^{24}$

The Kohn-Sham equations are solved in most cases within the full-potential Korringa-Kohn-Rostoker (KKR) Green function method ${ }^{25}$ with exact treatment of the atomic cell shapes. ${ }^{26}$ We took an angular momentum cutoff of $l_{\max }=3$ for the Green function and $l_{\max }^{\mathrm{pot}}=2 l_{\max }=6$ for the potential and chargedensity expansion, and an integration mesh of $30 \times 30 \times 30$ points in the full Brillouin zone. Equal-volume Wigner-Seitz cells were used for all types of atoms with an inscribed sphere radius of $\sqrt{2} a / 4=1.999 \AA$. The substitutional disorder was described within the coherent potential approximation (CPA). For the calculation of magnetically noncollinear states and static magnon spectra we employed the full-potential linearized augmented plane-wave $e^{27-29}$ (FLAPW) method as implemented in the FLEUR code,$^{30}$ using a plane-wave cutoff of $k_{\max }=4$ a.u. $^{-1}$, an angular momentum cutoff of $l_{\max }=8$, muffin-tin radii of $1.19 \AA$ for $\mathrm{Fe}$ and $\mathrm{Mn}$ and $1.222 \AA$ for $\mathrm{Si}$, and a $17 \times 17 \times 17 \mathrm{k}$-point mesh in the full Brillouin zone. The FLAPW code was also used to cross check the KKR results in some cases. Relativistic effects were taken into consideration within the scalar relativistic approximation, whereas spin-orbit coupling was not accounted for.

Since the lattice parameter varies only slightly ${ }^{9}$ in the range $0 \leqslant x \leqslant 1$ we used the experimental value at $x=0, a=$ $5.653 \AA$, in all calculations.

Magnetic excitations are modeled within a classical Heisenberg Hamiltonian,

$$
\begin{aligned}
H & =-\sum_{i j} J_{i j} \hat{e}_{i} \cdot \hat{e}_{j}, \\
& =-\sum_{i j} \tilde{J}_{i j} \vec{M}_{i} \cdot \vec{M}_{j},
\end{aligned}
$$

where $\hat{e}_{i}$ and $\hat{e}_{j}$ are unit vectors along the directions of the spin moments, $\vec{M}_{i}$ and $\vec{M}_{j}$, at sites $i$ and $j$, while the exchange pair-interaction constants $J_{i j}$ reflect the energy cost for the mutual tilting of the moments. It is sometimes convenient for the discussion to use form (2) with $\tilde{J}_{i j}=J_{i j} /\left(M_{i} M_{j}\right)$. The constants $J_{i j}$ were extracted from the spin-dependent KKR structural Green functions $G_{i j}^{\uparrow(\downarrow)}(E)$ and $t$ matrices $t_{i}^{\uparrow(\downarrow)}(E)$ by virtue of the Liechtenstein formula, ${ }^{31}$

$$
J_{i j}=\frac{1}{4 \pi} \operatorname{Im} \operatorname{Tr} \int^{E_{\mathrm{F}}} G_{i j}^{\uparrow}\left(t_{j}^{\uparrow}-t_{j}^{\downarrow}\right) G_{j i}^{\downarrow}\left(t_{i}^{\uparrow}-t_{i}^{\downarrow}\right) d E .
$$

Here, $G_{i j}^{\uparrow(\downarrow)}(E)$ and $t_{i}^{\uparrow(\downarrow)}(E)$ are matrices in angular momentum space and $\operatorname{Tr}$ denotes a trace over the angular momentum indices $(l m)$.

Having calculated the exchange constants, the Curie temperature of the compounds was calculated within a Monte Carlo method. For this purpose, exchange constants of atom pairs $(i, j)$ with distance up to 2.18 lattice constants were used; the simulation supercells included 1536 magnetic atoms (512 unit cells). The Curie temperature was identified through the characteristic peak of the calculated susceptibility. The method, either in combination with Monte Carlo simulations or with the random-phase approximation for the solution of the Heisenberg model, has proven useful for the calculation of the exchange constants and Curie temperature with a 10-15\% accuracy in several cases, including elemental ferromagnets and intermetallic alloys. ${ }^{32-34}$

\section{GROUND-STATE MAGNETIC MOMENTS AND CONFIGURATION}

The calculated magnetic moments (per atom and total) as a function of concentration are depicted in Fig. 2. Here the Mn atoms were assumed to reside at the $B$ site for all $x$. Evidently, the $B$-site atoms ( $\mathrm{Fe}$ and $\mathrm{Mn}$ alike) are generally in a high spin state, while the $\mathrm{Fe}$ atoms at the $A$ and $C$ 


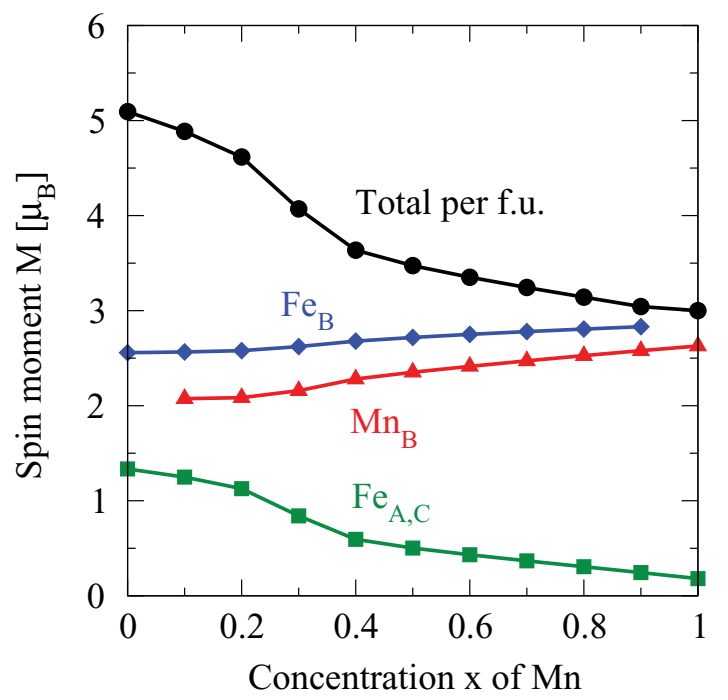

FIG. 2. (Color online) Calculated magnetic moments per atom and total magnetic moment in the unit cell as a function of $\mathrm{Mn}$ concentration in $\mathrm{Fe}_{3-x} \mathrm{Mn}_{x} \mathrm{Si}$.

sites are in a low-spin state. A small, monotonic increase of the $B$-site atomic moment is observed as a function of the Mn concentration: the $\mathrm{Fe}_{\mathrm{B}}$ moment ranges between $2.6 \mu_{\mathrm{B}}$ and $2.9 \mu_{\mathrm{B}}$, while the Mn moment ranges between $2.1 \mu_{\mathrm{B}}$ and $2.6 \mu_{\mathrm{B}}$. In strong contrast, the $\mathrm{Fe}_{\mathrm{A}, \mathrm{C}}$ moments drop significantly as the Mn content increases, from $1.3 \mu_{\mathrm{B}}$ for pure $\mathrm{Fe}_{3} \mathrm{Si}$ to $0.2 \mu_{\mathrm{B}}$ for the ordered $\mathrm{Fe}_{2} \mathrm{MnSi}$ alloy. This drop of the $\mathrm{Fe}_{\mathrm{A}, \mathrm{C}}$ moment causes the decrease in the total magnetic moment per unit cell.

This trend is in agreement with experimental findings ${ }^{2,8,12}$ that the average moment at the $B$ site is high and remains more or less unaffected by Mn doping, while the $A$ - and $C$-site moments drop significantly. (A deviation from the experimental result is found for $x \geqslant 0.8$, where there is experimental evidence for re-entrant behavior and reordering of spins.) The trend can be understood by an analysis of the density of states and an understanding of the different wave-function hybridization of $\mathrm{Mn}_{\mathrm{B}}-\mathrm{Fe}_{\mathrm{A}, \mathrm{C}}$ and $\mathrm{Fe}_{\mathrm{B}}-\mathrm{Fe}_{\mathrm{A}, \mathrm{C}}$ atoms; we defer the discussion to Sec. V.

The charges in the Wigner-Seitz cells differ only slightly from neutrality. The $\mathrm{Fe}_{\mathrm{A}, \mathrm{C}}$ atoms carry an excess electron charge, compensated mainly by the $\mathrm{Si}$ atom. The results are summarized in Table I.

In the calculation, the $\mathrm{Mn}$ dopants can be chosen to align ferromagnetically or antiferromagnetically to the $\mathrm{Fe}_{3} \mathrm{Si}$ matrix. Total-energy calculations are then needed to identify the correct ground state, which experimentally is found to be ferromagnetic, ${ }^{12}$ at least for concentrations $x<0.75$. The

TABLE I. Excess charge per atom (in units of $|e|$ ) in the WignerSeitz cells at different concentrations.

\begin{tabular}{lcccc}
\hline \hline$x$ & $\mathrm{Fe}_{\mathrm{A}, \mathrm{C}}$ & $\mathrm{Fe}_{\mathrm{B}}$ & $\mathrm{Mn}$ & $\mathrm{Si}$ \\
\hline 0.1 & -0.15 & 0.02 & 0.06 & 0.29 \\
0.5 & -0.18 & 0.06 & 0.10 & 0.28 \\
0.9 & -0.19 & 0.09 & 0.12 & 0.27 \\
\hline \hline
\end{tabular}

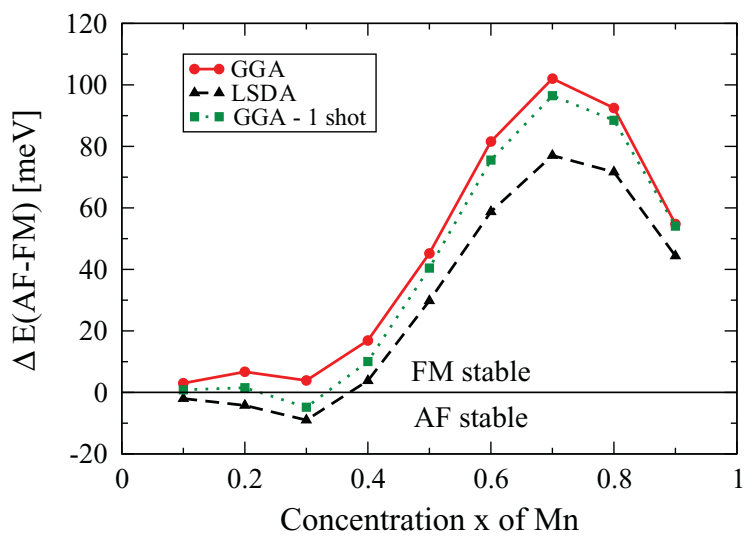

FIG. 3. (Color online) Results of total-energy calculations on the magnetic state of $\mathrm{Fe}_{3-x} \mathrm{Mn}_{x} \mathrm{Si}$. The energy difference $\Delta E=$ $E(\mathrm{AF})-E(\mathrm{FM})$ between the antiferromagnetic and the ferromagnetic alignment of $\mathrm{Mn}$ atoms with respect to the $\mathrm{Fe}_{\mathrm{B}}$ atoms is shown ( $\Delta E$ represents here values per unit cell). The GGA predicts the correct ferromagnetic state, while the LSDA yields an antiferromagnetic orientation of the Mn moments with respect to the $\mathrm{Fe}_{3} \mathrm{Si}$ matrix at low concentrations, which turns to ferromagnetic at high concentrations. A single-shot GGA calculation improves the LSDA result somewhat, but not quite.

calculations within the GGA show that the ferromagnetic state is stable for all $\mathrm{Mn}$ concentrations $0<x \leqslant 1$ (see Fig. 3). However, the result within the LSDA is that the antiferromagnetic state is more stable than the ferromagnetic one for $x \lesssim 0.35$, in clear disagreement with experiment; ${ }^{12}$ actually, for $0.2 \lesssim x \lesssim 0.8$, the LSDA lowest energy is found in a disordered local moment state of the form $\mathrm{Fe}_{3-x} \mathrm{Mn}_{x-y}^{\downarrow} \mathrm{Mn}_{y}^{\uparrow} \mathrm{Si}$, as the $\mathrm{Mn}$ atoms progressively change the moment orientation from antiferromagnetic $\left(\mathrm{Mn}^{\downarrow}\right)$ to ferromagnetic $\left(\mathrm{Mn}^{\uparrow}\right)$. This indicates a possible noncollinear LSDA ground state (our calculations for the disordered alloys were always magnetically collinear). The dispute between the LSDA and GGA results at low concentrations was cross checked and verified by a calculation within the FLAPW method, where a low Mn concentration of $x=0.125$ was approximated by construction of a large supercell (the same lattice parameter was used in LSDA and GGA calculations; see Sec. III).

The failure of the LSDA to predict the correct magnetic ground state can be attributed to the exchange and correlation part of the total energy, rather than the single-particle energies. We arrive at this conclusion for two reasons. First, the GGA density of states is very similar to the LSDA density of states (when both are calculated in the ferromagnetic configuration). Second, we attested our suggestion by using the single-shot GGA (described in Sec. III), which changes only the exchange-correlation part of the total energy, while retaining the LSDA single-particle energies. The total-energy results are then improved significantly, although not entirely, toward the correct magnetic ground state as can be seen in Fig. 3. We note that there is no general rule favoring the GGA over the LSDA as far as the magnetic properties are concerned. For example, long-wavelength spin-wave spectra calculated within the adiabatic approximation agree rather well with experiment if the LSDA is used (see, e.g., Pajda et al. ${ }^{35}$ for $\mathrm{Fe}, \mathrm{Co}$, and $\mathrm{Ni}$, or Buczek et al ${ }^{36}$ for intermetallic alloys). 
There are even reported cases, such as $\mathrm{Fe}_{3} \mathrm{Al}$, ${ }^{37}$ where GGA gives the wrong crystal structure, while LSDA corrects the structure as well as the magnetic moments.

\section{DENSITY OF STATES, MAGNETIC MOMENTS, AND HALF METALLIC BEHAVIOR}

\section{A. Trends of DOS with concentration; magnetic moments}

The density of states (DOS) of $\mathrm{Fe}_{3-x} \mathrm{Mn}_{x} \mathrm{Si}$ at various concentrations $x$ is depicted in Fig. 4. The gross features have been analyzed in the past in studies of full Heusler alloys. ${ }^{38}$ Most important points specifically for our discussion are as follows: (i) The hybridization of $d$ states of $t_{2 g}$ character of the $B$-site atom (Fe or $\mathrm{Mn}$ ) with $t_{2 g}$ states of the $A$ - and $C$-site Fe atoms. (ii) The strong-ferromagnet character of the $B$ atoms as opposed to the weak-ferromagnet character of the $(A, C)$ atoms. (iii) The progressive shift, for charge-neutrality reasons, of the majority-spin states at the $B$ site as the $\mathrm{Mn}$ concentration is increased, dragging with them the $(A, C)$ site $t_{2 g}$ states due to hybridization and affecting the $\mathrm{Fe}_{\mathrm{A}, \mathrm{C}}$ moment and DOS. We now discuss these points in detail; the trends of the magnetization and the appearance of half-metallicity are directly connected to this behavior and also discussed in this section.

(i) The $d$ states of $\mathrm{Fe}_{\mathrm{B}}$ and $\mathrm{Mn}$ are split by the octahedral environment into two irreducible subspaces: the $t_{2 g}$, including the $d_{x y}, d_{y z}$, and $d_{x z}$ orbitals, and the $e_{g}$, including the $d_{x^{2}-y^{2}}$ and $d_{z^{2}}$ orbitals $(x, y$, and $z$ are implied to be directions along the cubic crystal axes). The $\mathrm{Fe}_{\mathrm{A}, \mathrm{C}} d$ states are also split by the tetrahedral environment. However, it is also important that $d$ orbitals of $\mathrm{Fe}_{\mathrm{A}, \mathrm{C}}$ atoms interact with each other, although the $\mathrm{Fe}_{\mathrm{A}, \mathrm{C}}$ atoms are second-nearest neighbors. This interaction produces bonding-antibonding splittings between the $t_{2 g}$ and $t_{1 u}$ type states, and also between $e_{g}$ and $e_{u}$ type states. ${ }^{38}$

Hybridization between $d$ states of $B$-site atoms with $\mathrm{Fe}_{\mathrm{A}, \mathrm{C}}$ atoms is allowed only among the states of $t_{2 g}$ or the ones of $e_{g}$ character; by symmetry, the $t_{1 u}$ and $e_{u}$ states remain oblivious to the $d$ states of their $B$ site neighbors. ${ }^{38}$ The symmetry-decomposed DOS is shown in Fig. 5 for $\mathrm{Fe}_{3} \mathrm{Si}$, and is completely analogous in $\mathrm{Fe}_{3-x} \mathrm{Mn}_{x} \mathrm{Si}$, with the peaks appropriately shifted as discussed below.

(ii) On the one hand, the local DOS of the $B$-site atoms (either $\mathrm{Fe}$ or $\mathrm{Mn}$ ) has in all cases the characteristics of the DOS of a strong ferromagnet. Namely, the $B$-site local DOS of one spin direction (here spin down) is very low at $E_{\mathrm{F}}$ and in a region around $E_{\mathrm{F}}$, as is evident by inspection of Fig. 4. Consequently, on-site transfer between spin-up and spin-down charge is energetically expensive, because a strong shift of $d$ bands is involved. This, together with the requirement of local charge neutrality in a metallic system, stabilizes the local atomic moment of $\mathrm{Fe}$ or $\mathrm{Mn}$ at the $B$ site against perturbations (such as change of concentration or
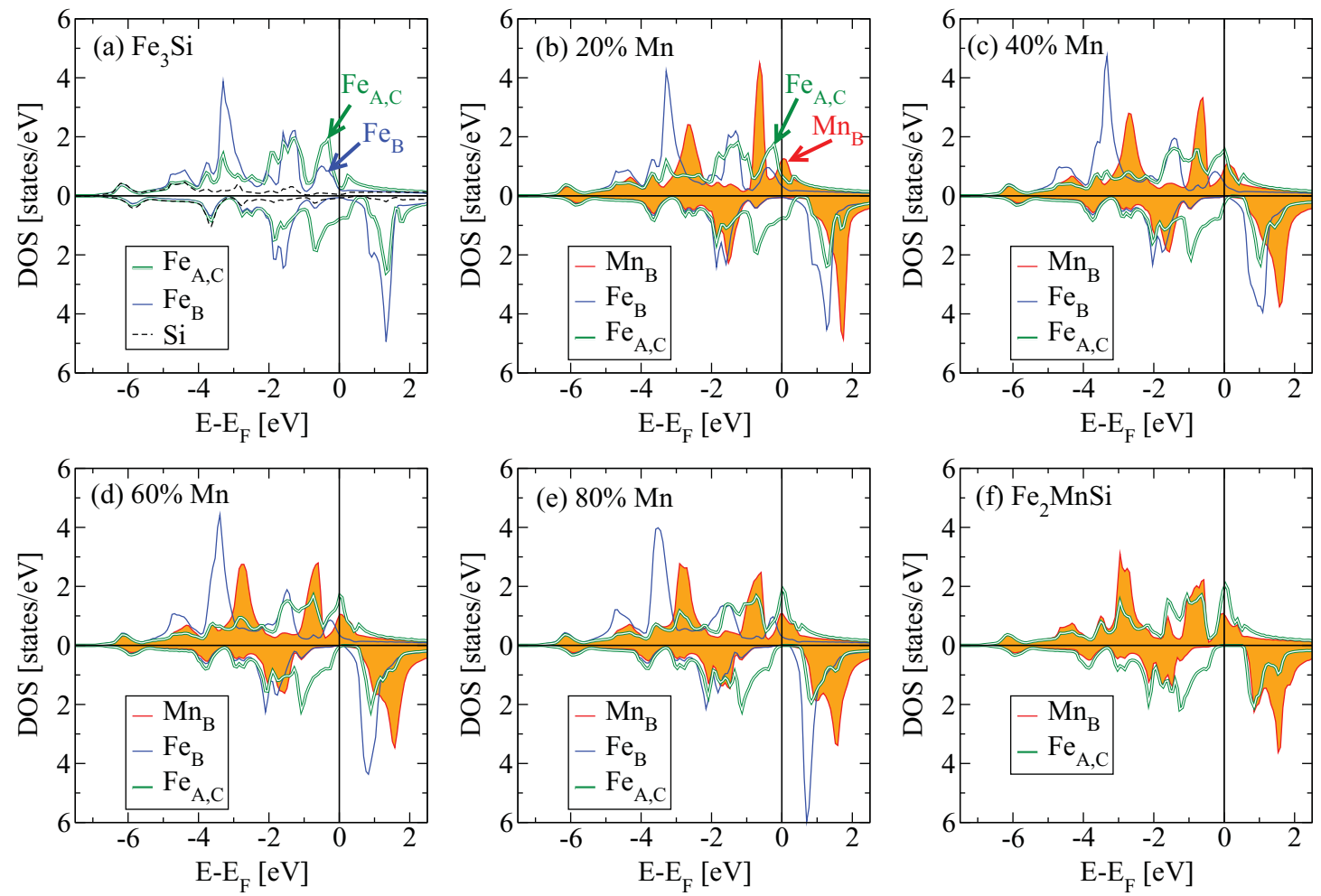

FIG. 4. (Color online) Spin- and atom-resolved density of states of $\mathrm{Fe}_{3-x} \mathrm{Mn}_{x} \mathrm{Si}$ alloys for $\mathrm{Mn}$ concentrations $0 \leqslant x \leqslant 1$. In all cases, the $\mathrm{Mn}$ atom taken to reside at the $B$ site. In (a) and (b), arrows indicate the positions of the DOS peaks near $E_{\mathrm{F}}$ which are responsible for the moment trends, as discussed in the text. The calculations were done within the Korringa-Kohn-Rostoker (KKR)-CPA. In each plot, the upper panel corresponds to spin-up (majority-spin) DOS and the lower panel (with inverted ordinate) to spin-down (minority-spin) DOS. The shaded area (orange area inside the red lines) corresponds to $\mathrm{Mn}$, the full blue line to $\mathrm{Fe}_{\mathrm{B}}$, and the double green line to $\mathrm{Fe}_{\mathrm{A}, \mathrm{C}}$. 


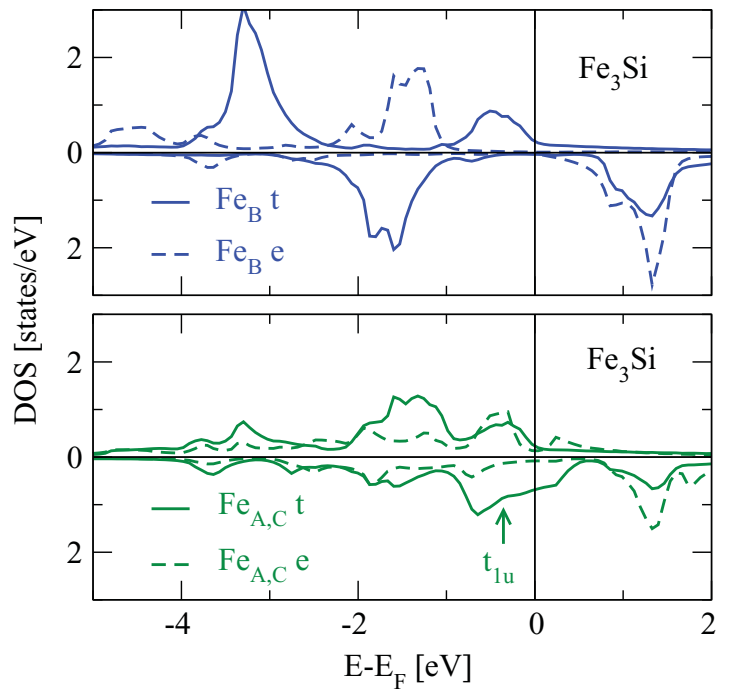

FIG. 5. (Color online) Symmetry-resolved local density of states of $\mathrm{Fe}_{3} \mathrm{Si}$ at the $\mathrm{Fe}_{\mathrm{B}}$ (top) and $\mathrm{Fe}_{\mathrm{A}, \mathrm{C}}$ (bottom) atoms. In each plot, the upper panel corresponds to spin-up and the lower panel to spin-down DOS.

lattice parameter). Therefore there is only weak dependence of the $\mathrm{Fe}$ and $\mathrm{Mn}$ moment on concentration, as seen in Fig. 2 . The strong-ferromagnet behavior of the $B$ site is favored by its octahedral bcc-like coordination, which results in large bonding-antibonding splittings via $t_{2 g}-t_{2 g}$ and $e_{g}-e_{g}$ hybridization of the $B$-site atoms with $\mathrm{Fe}_{\mathrm{A}, \mathrm{C}}$ neighbors. The line shape of the local DOS at the $B$ site is reminiscent of bcc iron.

On the other hand, the local DOS of the $\mathrm{Fe}_{\mathrm{A}, \mathrm{C}}$ atoms has the characteristics of a weak ferromagnet, i.e., $d$ states of both spin directions are present at and around $E_{\mathrm{F}}$. This allows for energetically cheap transfer between spin-up density and spindown density, and therefore relatively easy change of moment. Note from Fig. 2 that the drop of the $\mathrm{Fe}_{\mathrm{A}, \mathrm{C}}$-moment is strongest for $0.2<x<0.4$, at the same concentration range when the $\mathrm{Fe}_{\mathrm{A}, \mathrm{C}} \mathrm{DOS}$ at $E_{\mathrm{F}}$ is large for both spins in Fig. 4, i.e., when the weak-ferromagnet character is most evident; before $x=0.2$ there is a DOS valley at $E_{\mathrm{F}}$ for spin up, and after $x=0.4$ there is a valley for spin down. The weak-ferromagnet behavior of $\mathrm{Fe}_{\mathrm{A}, \mathrm{C}}$ is favored by the $t_{1 u}$ and $e_{u}$ states forming the spin-down peak of $\mathrm{Fe}_{\mathrm{A}, \mathrm{C}}$ around $E_{\mathrm{F}}$ in $\mathrm{Fe}_{3} \mathrm{Si}$.

(iii) Now we consider the consequences of observations (i) and (ii) (see Fig. 6). We first focus on the $B$ site in $\mathrm{Fe}_{3-x} \mathrm{Mn}_{x} \mathrm{Si}$. Since a metal must show approximate local charge neutrality, the Mn states must be appropriately shifted with respect to those of $\mathrm{Fe}$ so that one less electron is accommodated by Mn. This is achieved by a shift of the spin-up $t_{2 g}$ and $e_{g}$ peaks, which are just under $E_{\mathrm{F}}$ for the $\mathrm{Fe}_{\mathrm{B}}$ atom, so that they fall at $E_{\mathrm{F}}$ for the $\mathrm{Mn}$ atom [as indicated by arrows in Figs. 4(a) and 4(b)]. Now, there is a peak of $t_{2 g}$ character [also indicated by arrows in Figs. 4(a) and 4(b)] in the $\mathrm{Fe}_{\mathrm{A}, \mathrm{C}}$ DOS, associated by hybridization to the aforementioned $B$-site peak. This, at low Mn concentrations, is associated more to the $\mathrm{Fe}_{\mathrm{B}}$ DOS, while at high Mn concentrations it is associated more to the Mn DOS. At intermediate concentrations, as the $\mathrm{Mn}$ content increases, the $\mathrm{Fe}_{\mathrm{A}, \mathrm{C}}$ peak is dragged to higher energies and starts crossing the Fermi level, depriving the

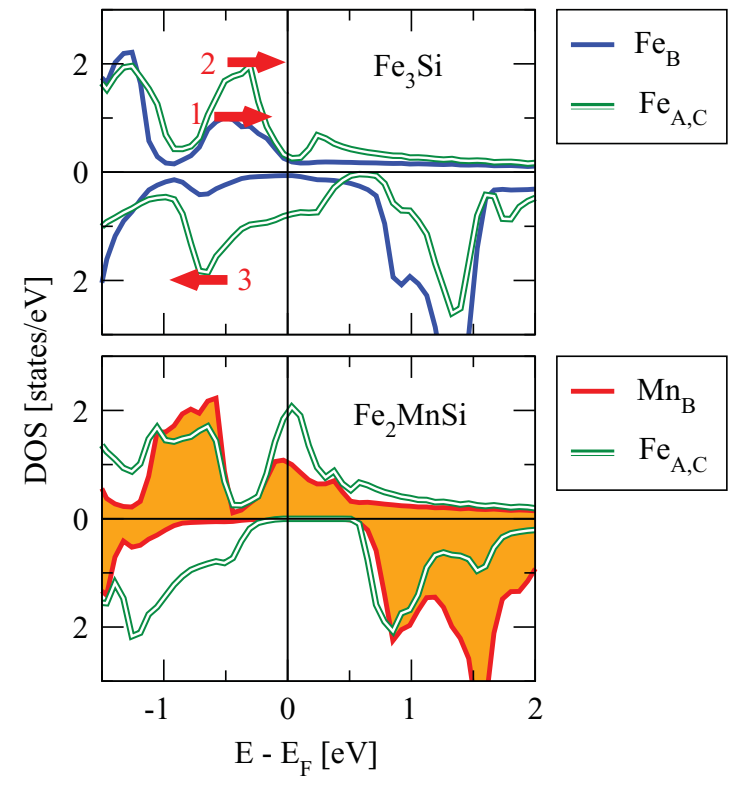

FIG. 6. (Color online) Spin- and atom-resolved density of states of $\mathrm{Fe}_{3} \mathrm{Si}$ (top) and $\mathrm{Fe}_{2} \mathrm{MnSi}$ (bottom) around $E_{\mathrm{F}}$. Arrows indicate the shift of the peaks upon substitution of Fe by $\mathrm{Mn}$ that lead to the reduction of the moment and half metallic behavior. 1: The $B$-site spin-up peak shifts higher in order to dispose of one electron. 2: The spin-up $\mathrm{Fe}_{\mathrm{A}, \mathrm{C}}$ peaks are dragged along due to hybridization, disposing of spin-up charge also at the $\mathrm{Fe}_{\mathrm{A}, \mathrm{C}}$ atoms. 3: The spin-down peak at $\mathrm{Fe}_{\mathrm{A}, \mathrm{C}}$ must shift lower in order to maintain local charge neutrality. The result is a reduction of the magnetic moment and an increase of the spin polarization at $E_{\mathrm{F}}$. In each plot, the upper panel corresponds to spin-up and the lower panel to spin-down DOS. The orange (shaded) area inside the red line corresponds to $\mathrm{Mn}$, the full blue line to $\mathrm{Fe}_{\mathrm{B}}$, and the double green line to $\mathrm{Fe}_{\mathrm{A}, \mathrm{C}}$.

$\mathrm{Fe}_{\mathrm{A}, \mathrm{C}}$ atoms of spin-up charge. This is readily compensated (to maintain local charge neutrality) by a shift of spin-down $\mathrm{Fe}_{\mathrm{A}, \mathrm{C}}$ $t_{1 u}$ states from $E_{\mathrm{F}}$ to slightly below $E_{\mathrm{F}}$, gaining spin-down charge. The net effect is a reduction of the $\mathrm{Fe}_{\mathrm{A}, \mathrm{C}}$ spin moment, accompanied by the appearance of a spin-down gap at $E_{\mathrm{F}}$, i.e., the signature of a half metallic behavior. Thus we see that the $t_{2 g}-t_{2 g}$ hybridization between states at the $B$ and $A, C$ sites, together with the requirement of local charge neutrality, leads to the drop of $\mathrm{Fe}_{\mathrm{A}, \mathrm{C}}$ moment as the $\mathrm{Mn}$ concentration increases.

In spite of the calculated drop of the magnetization per formula unit, the values are still too high compared to experiment, ${ }^{2}$ although they are in agreement with previous calculations. ${ }^{3,18,19,38}$ For $\mathrm{Fe}_{2} \mathrm{MnSi}$, the calculated value is $3 \mu_{\mathrm{B}}$, while the experiment gives $M_{0} \approx 1.5 \mu_{\mathrm{B}}$ and $M_{\text {extr }} \approx 2.3 \mu_{\mathrm{B}}$. We propose an explanation of this discrepancy in Sec. VII.

\section{B. Spin polarization and transition to half metallic behavior}

The spin polarization $P$ at $E_{\mathrm{F}}$ is defined as

$$
P=\frac{n_{\uparrow}\left(E_{\mathrm{F}}\right)-n_{\downarrow}\left(E_{\mathrm{F}}\right)}{n_{\uparrow}\left(E_{\mathrm{F}}\right)+n_{\downarrow}\left(E_{\mathrm{F}}\right)}
$$

with $n_{\uparrow}\left(E_{\mathrm{F}}\right)$ and $n_{\downarrow}\left(E_{\mathrm{F}}\right)$, respectively, the spin-up and spindown DOS at $E_{\mathrm{F}}$. The mechanism described in the previous subsection, involving a shift of the peaks around $E_{\mathrm{F}}$, leads to a 


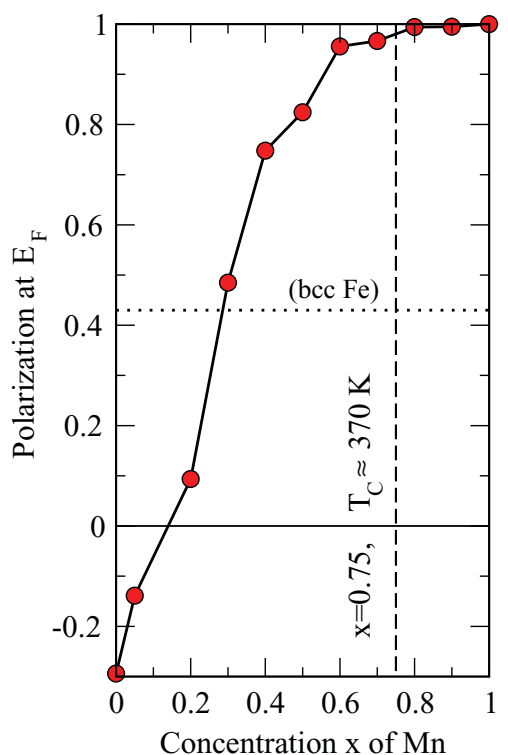

FIG. 7. (Color online) Calculated spin polarization $P$ at $E_{\mathrm{F}}$ as a function of Mn concentration $x$ in $\mathrm{Fe}_{3-x} \mathrm{Mn}_{x} \mathrm{Si}$. For $x \geqslant 0.7$ the alloy is half metallic. The polarization of bcc Fe (dotted line) is also shown for comparison.

drastic change of $P$ as a function of the Mn concentration. This is visualized in Fig. 7. At low $x, P$ is negative, approximately -0.3 (the negative sign means that the DOS at $E_{\mathrm{F}}$ is dominated by minority-spin carriers). At higher concentrations $P$ crosses zero and reaches high positive values, as the spin-up DOS peak shifts to $E_{\mathrm{F}}$, while the spin-down peak is retracted below $E_{\mathrm{F}}$. At around $x=0.75$ the spin polarization reaches the highest possible value of $P=1$, and the alloy becomes half metallic. At this point the (experimentally found) Curie temperature is about $370 \mathrm{~K}$. A half metallic behavior of ordered $\mathrm{Fe}_{2} \mathrm{MnSi}$ was also found in previous calculations. ${ }^{3,18,19,38}$

This is a rare occasion in which a continuous change of a material parameter-here the Mn concentration-results in a continuous change of the spin polarization over such an extended range. Assuming that this effect is present in experiment, it could be efficiently used to compare to each other various experimental methods of probing the spin polarization (such as spin-polarized photoemission spectroscopy, positron annihilation, Andreev reflection, or tunneling magnetoresistance). It should be noted that the half metallic behavior is already present at $x=0.75$, i.e., before the start of the re-entrant behavior.

\section{EXCHANGE INTERACTIONS AND CURIE TEMPERATURE}

The exchange pair-interaction constants $J_{i j}$ were calculated for several Mn concentrations as described in Sec. III. They are plotted as a function of distance in Fig. 8. In all cases, we find that the dominant contribution comes from the firstneighbor interaction $J_{1}$ between the site- $B$ atom ( $\mathrm{Mn}$ or $\mathrm{Fe}$ ) and $\mathrm{Fe}_{\mathrm{A}, \mathrm{C}}$. The $\mathrm{Fe}_{\mathrm{B}}-\mathrm{Fe}_{\mathrm{A}, \mathrm{C}}$ interaction, $J_{1}(\mathrm{Fe}-\mathrm{Fe})$, is between 20 and $10 \mathrm{meV}$, depending on concentration, and the $\mathrm{Mn}_{\mathrm{B}}-\mathrm{Fe}_{\mathrm{A}, \mathrm{C}}$ interaction, $J_{1}(\mathrm{Mn}-\mathrm{Fe})$, is between 3 and $5 \mathrm{meV}$; next-nearestneighbor interactions ( $\mathrm{Fe}-\mathrm{Fe}, \mathrm{Mn}-\mathrm{Mn}$ or $\mathrm{Fe}-\mathrm{Mn}$ ) are typically

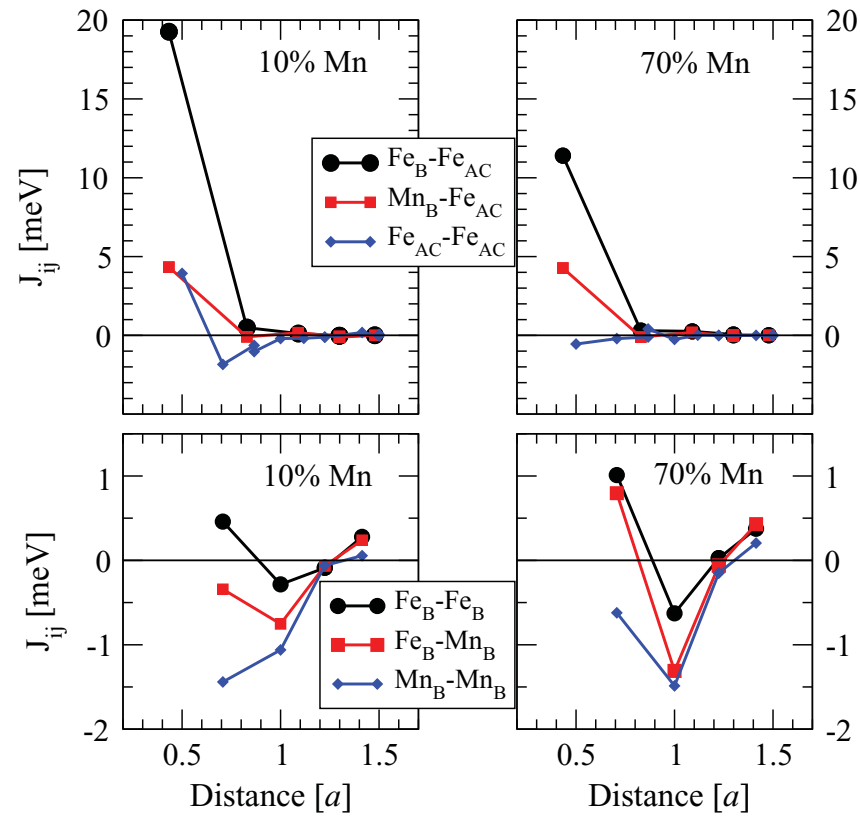

FIG. 8. (Color online) Pair exchange constants as a function of the interatomic distance. Left: $10 \% \mathrm{Mn}$ concentration. Right: $70 \%$ Mn concentration. Note the different energy scale in the upper and lower panels. The lines are guides to the eye.

at least one order of magnitude weaker. Therefore we expect the Curie temperature trend with concentration to follow the behavior of the averaged $J_{1}$, at least qualitatively.

The trends of $J_{1}$ with concentration are depicted in Fig. 9, together with the moment of the $\mathrm{Fe}_{\mathrm{A}, \mathrm{C}}$ atoms, $M\left(\mathrm{Fe}_{\mathrm{A}, \mathrm{C}}\right)$. There is a clear correlation between $J_{1}\left(\mathrm{Fe}_{\mathrm{B}}-\mathrm{Fe}_{\mathrm{A}, \mathrm{C}}\right)$ and $M\left(\mathrm{Fe}_{\mathrm{A}, \mathrm{C}}\right)$, while $J_{1}\left(\mathrm{Mn}-\mathrm{Fe}_{\mathrm{A}, \mathrm{C}}\right)$ seems unaffected by the drop of $M\left(\mathrm{Fe}_{\mathrm{A}, \mathrm{C}}\right)$. We now discuss these observations. The pairinteraction energies $E_{i j}=-J_{i j} \hat{e}_{i} \cdot \hat{e}_{j}$, determined by the electronic structure, contain the absolute value of the atomic spin moments in a nontrivial way. By this we mean that, if the moments are varied by some external parameter ( e.g., here,

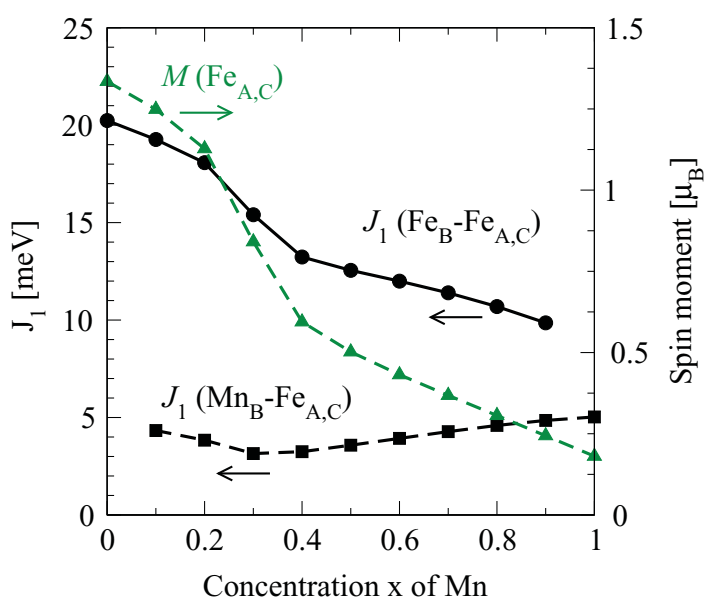

FIG. 9. (Color online) Nearest-neighbor exchange interactions $J_{1}$ between $\mathrm{Fe}_{\mathrm{B}}$ and $\mathrm{Fe}_{\mathrm{A}, \mathrm{C}}$ atoms and between $\mathrm{Mn}_{\mathrm{B}}$ and $\mathrm{Fe}_{\mathrm{A}, \mathrm{C}}$ atoms, as calculated by the Liechtenstein formula (3). The $\mathrm{Fe}_{\mathrm{A}, \mathrm{C}}$ moments, $M\left(\mathrm{Fe}_{\mathrm{A}, \mathrm{C}}\right)$, are also shown, to demonstrate the correlation between the decrease of $J_{1}$ and $M\left(\mathrm{Fe}_{\mathrm{A}, \mathrm{C}}\right)$. 
by changing the concentration), $E_{i j}$ can be affected either just by the variation of the absolute value of the moment [as suggested by the alternative form derived from Eq. (2), $\left.E_{i j}=-\tilde{J}_{i j} \vec{M}_{i} \cdot \vec{M}_{j}\right]$, or also by an alteration of the exchange mechanism, which is induced by the change of the electronic structure via the external parameter and affects the constants $J_{i j}$.

Apparently, in $\mathrm{Fe}_{3-x} \mathrm{Mn}_{x} \mathrm{Si}$ we are faced with both situations. On the one hand, the dominant trend for $J_{1}(\mathrm{Fe}-\mathrm{Fe})$ comes from the reduction of the $\mathrm{Fe}_{\mathrm{A}, \mathrm{C}}$ moment as the Mn concentration $x$ increases, although there seems to be also an alteration of $\tilde{J}_{1}$, since the drop of $M\left(\mathrm{Fe}_{\mathrm{A}, \mathrm{C}}\right)$ is faster than the drop of $J_{1}$. On the other hand, $J_{1}(\mathrm{Mn}-\mathrm{Fe})$ is left practically unaltered despite the strong reduction of $M\left(\mathrm{Fe}_{\mathrm{A}, \mathrm{C}}\right)$. In order to interpret this, we again observe the shifting of the peak, indicated by an arrow in the $\mathrm{Fe}_{\mathrm{A}, \mathrm{C}}$ DOS in Fig. 4, as a function of $x$. This hybridization-induced peak coincides more and more with its associated Mn peak as the Mn concentration is increased. Since the Fermi level bisects the Mn peak (and increasingly more the $\mathrm{Fe}_{\mathrm{A}, \mathrm{C}}$ peak), the double-exchange mechanism sets in progressively more and more, favoring ferromagnetic alignment of the moments. (We remind the reader that the double-exchange mechanism is present when half filled states of the same spin hybridize with each other, resulting in a band broadening and a gain in energy; in a tight-binding picture, the kinetic energy is lowered by the interatomic hopping of electrons, allowed by the half filled band.) Thus the progressive shift of the indicated peak in the $\mathrm{Fe}_{\mathrm{A}, \mathrm{C}}$ DOS causes two competing effects: a reduction of the moment $M\left(\mathrm{Fe}_{\mathrm{A}, \mathrm{C}}\right)$ (as discussed in Sec. V) and a strengthening of the Mn-Fe pair exchange interaction. These effects by and large cancel each other in the Heisenberg energy expression, and the net result is only a weak dependence of $J_{1}(\mathrm{Mn}-\mathrm{Fe})$ on concentration.

We close the discussion on the exchange parameters with the following comments on the calculations. In the present work, the exchange constants were calculated starting from the ground-state (ferromagnetic) configuration. As a test, however, we calculated the Mn-Fe exchange constants starting from the antiferromagnetic (AF) state (i.e., with the Mn moments antiferromagnetically aligned to the $\mathrm{Fe}_{3} \mathrm{Si}$ matrix) for a Mn concentration of $x=0.1$, and compared with the result starting from the ferromagnetic (FM) ground state. As can be seen in Fig. 10, there is a strong qualitative difference in the calculated value of the nearest-neighbor Mn-Fe interaction in the two cases: Starting from FM, we obtain a tendency to retain ferromagnetism (positive exchange constant); while starting from the AF state, we obtain a tendency to retain antiferromagnetism (negative exchange constant). For the more distant neighbors the two calculations give quantitative, but not so much qualitative, differences. This discrepancy demonstrates the significant change in electronic structure at large angles, far beyond the assumptions of a Heisenberg model. The discrepancy is not observed at higher Mn concentrations, when the electronic structure of the $\mathrm{Fe}_{\mathrm{B}}$ atoms at $E_{\mathrm{F}}$ is dictated more and more by the hybridization of their $d$ states with the Mn $d$ states. In addition, we note that, in the case of $\mathrm{Fe}_{2} \mathrm{MnSi}$, the LSDA result for the $\mathrm{Mn}-\mathrm{Fe}_{\mathrm{A}, \mathrm{C}}$ nearest-neighbor exchange parameter is weaker by approximately $1 / 3$ compared to the

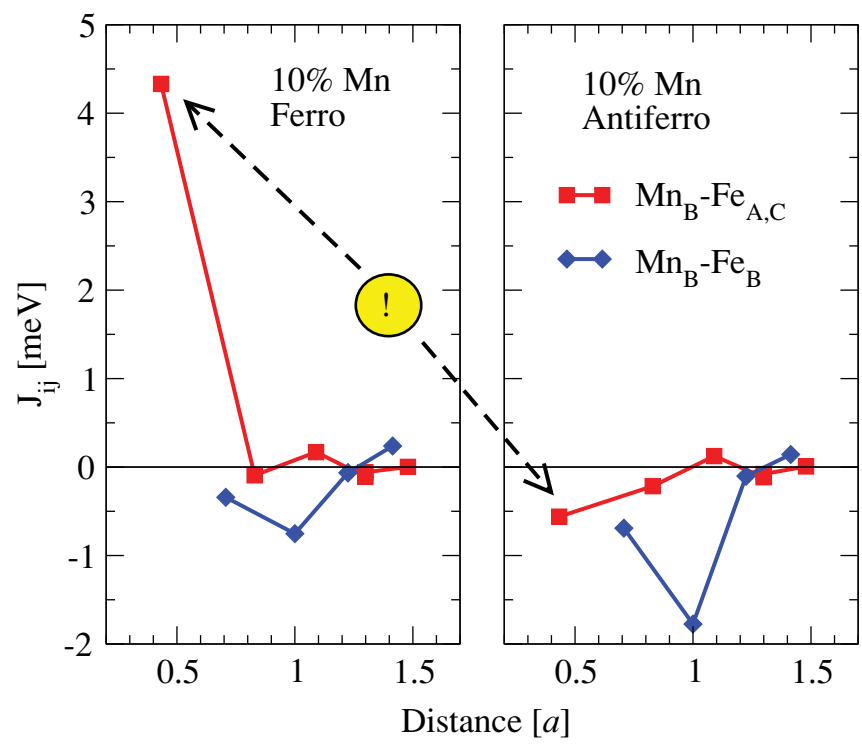

FIG. 10. (Color online) Calculated Mn-Fe exchange interaction $J_{i j}$ via the Liechtenstein formula (3) for a Mn concentration of $x=0.1$, starting from two different configurations. Left: ferromagnetic starting point (i.e., with the Mn moments ferromagnetically aligned to the $\mathrm{Fe}_{3} \mathrm{Si}$ matrix), which is also the ground state. Right: antiferromagnetic starting point (i.e., with the Mn moments antiferromagnetically aligned to the $\mathrm{Fe}_{3} \mathrm{Si}$ matrix). There is a strong qualitative difference in the dominant nearest-neighbor interaction, emphasized by an exclamation mark, while the more distant interactions change quantitatively, but not so much qualitatively. This discrepancy is not observed at higher Mn concentrations, when the electronic structure of the $\mathrm{Fe}_{\mathrm{A}, \mathrm{C}}$ atoms at $E_{\mathrm{F}}$ is dictated more by the hybridization of their $d$ states with the Mn $d$ states. The lines are guides to the eye.

GGA result. This reflects also the magnon spectra that are discussed in Sec. VII. Such a discrepancy between LSDA and GGA does not show up for $\mathrm{Fe}_{3} \mathrm{Si}$.

Calculated Curie temperatures $T_{\mathrm{C}}$ of $\mathrm{Fe}_{3-x} \mathrm{Mn}_{x} \mathrm{Si}$ are shown in Table II together with experimental results. The experimental finding of a reduction of $T_{\mathrm{C}}$ with increasing concentration is reproduced, although the calculated results systematically underestimate the experimental values. The reason for this trend is obviously the reduction of $J_{1}(\mathrm{Fe}-\mathrm{Fe})$ as a function of concentration, in conjunction with the comparatively low values of $J_{1}(\mathrm{Mn}-\mathrm{Fe})$ which become important at high concentrations. Note that, at $x=1$, the $\mathrm{Fe}_{2} \mathrm{MnSi}$ alloy is found experimentally ${ }^{10}$ to possess a degree of disorder in the form of a $\mathrm{Mn}_{\mathrm{B}}-\mathrm{Fe}_{\mathrm{A}, \mathrm{C}}$ swap of $12 \%$. When considering such a swap in the calculations, we found an increased $T_{\mathrm{C}}$ of $200 \mathrm{~K}$, mainly because the $\mathrm{Fe}$ atoms replacing $\mathrm{Mn}$ at the $B$ site

TABLE II. Calculated Curie temperature of $\mathrm{Fe}_{3-x} \mathrm{Mn}_{x} \mathrm{Si}$ at $x=0$, 0.5, and 1. Experimental results (Ref. 2) are also shown.

\begin{tabular}{lcc}
\hline \hline$x$ & $T_{\mathrm{C}}(\mathrm{K})$ (expt.) & $T_{\mathrm{C}}(\mathrm{K})($ calc. $)$ \\
\hline 0.0 & 803 & 730 \\
0.5 & 450 & 320 \\
1.0 & 200 & 160 (ordered; Mn at $B)$ \\
& & $200(12 \%$ Mn at $A, C)$ \\
\hline \hline
\end{tabular}




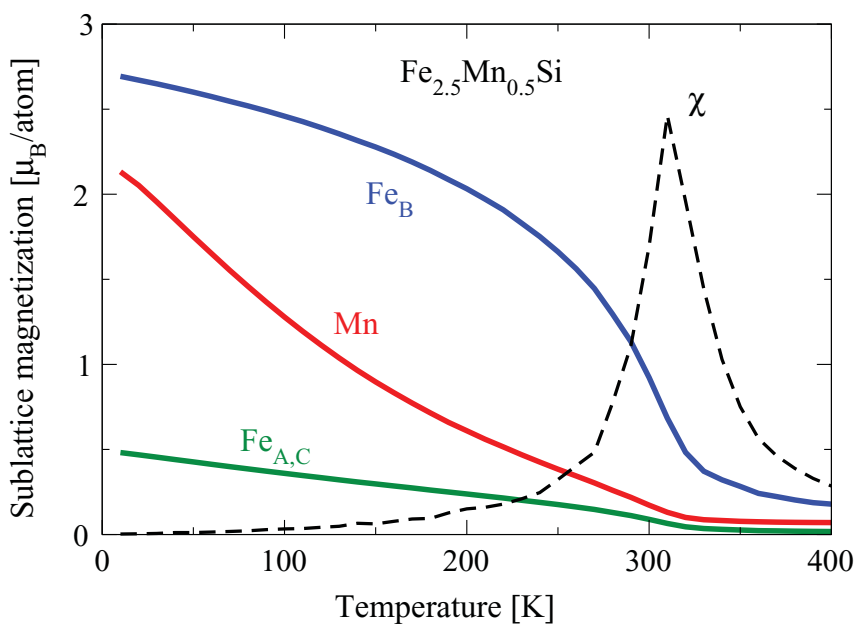

FIG. 11. (Color online) Monte Carlo simulation of the magnetization and susceptibility $\chi$ vs temperature for $\mathrm{Fe}_{2.5} \mathrm{Mn}_{0.5} \mathrm{Si}$. It is evident that the Mn sublattice magnetization drops much faster than the $\mathrm{Fe}$ magnetization. The susceptibility peak signals the Curie temperature.

have a stronger exchange interaction with the $\mathrm{Fe}_{\mathrm{A}, \mathrm{C}}$ neighbors. However, a possible Mn clustering at this concentrations, which could affect the value of the exchange interactions, cannot be taken into account within the CPA. This is discussed in more detail in the next section.

Before closing this section, we show an interesting behavior of the Mn sublattice magnetization at low or intermediate concentrations. Kepa et al. ${ }^{39}$ and Ersetz et al. ${ }^{40}$ find experimentally that the $\mathrm{Mn}_{\mathrm{B}}$ moment at room temperature is significantly lower than what is reported close to $T=0 \mathrm{~K}$. E.g., for $x=0.55,{ }^{40} M(\mathrm{Mn})=1.42 \mu_{\mathrm{B}}$ at room temperature instead of $2.2 \mu_{\mathrm{B}}$. Therefore they propose that the Mn average moment drops with temperature faster than the Fe moment. This is reproduced by our Monte Carlo simulations, and is due to the weak coupling of $\mathrm{Mn}$ to $\mathrm{Fe}_{\mathrm{A}, \mathrm{C}}$ compared to the coupling of $\mathrm{Fe}_{\mathrm{B}}$ to $\mathrm{Fe}_{\mathrm{A}, \mathrm{C}}$. As we see in Fig. 11, the Mn magnetization curve does not follow the critical-behavior form with an inflection point of the Fe curve, but rather drops almost linearly. This is also reflected in the Mn sublattice susceptibility (not shown here), which does not show a peak at $T=T_{\mathrm{C}}$.

\section{DISCUSSION ON THE RE-ENTRANT BEHAVIOR; LIMITATIONS OF PRESENT CALCULATIONS}

In Sec. II we summarized what is known on the anomalous behavior of the magnetization of $\mathrm{Fe}_{3-x} \mathrm{Mn}_{x} \mathrm{Si}$ for $x>0.75$. The magnetization $M(T)$ increases for $0<T<T_{\mathrm{R}}$ (contrary to the behavior in a usual ferromagnet), then decreases again up to $T_{\mathrm{C}} ; T_{\mathrm{R}}$ varies with concentration, starting from low values at $x=0.75$ and saturating at about $70-80 \mathrm{~K}$ at $x=1$. This so-called re-entrant behavior and the resulting re-ordered phase, which has been the focus of many experimental works, could not be reproduced by calculations within the approximations used in the present work.

There is experimental evidence, ${ }^{2,10}$ based on neutronscattering data, that the re-entrant behavior arises from transverse ordering of the $\mathrm{Mn}_{\mathrm{B}}$ magnetic moments. This means that, in the ground state, the Mn moments are partly canted to the direction of average magnetization. As the temperature is increased, the Mn moments absorb energy by aligning their spins and thus the average magnetization increases.

Mohn and Supanetz ${ }^{17}$ performed first-principles (LSDAbased) calculations of noncollinear magnetic structures for spin spirals of several wave vectors $\vec{q}$. For ordered $\mathrm{Fe}_{2} \mathrm{MnSi}$ they found that the ferromagnetic state represents a local minimum, while a spin spiral of wave vector $\vec{q}=(2 \pi / a)(0.45,0.45,0.45)$ has a lower energy by about $0.75 \mathrm{mRyd}(10.2 \mathrm{meV})$. The absolute energy minimum which they found is very slightly lower, for a state which shows a canted antiferromagnetic ordering of the Mn moments along the [111] axis, with a canting angle of $60^{\circ}$ with respect to the [111] axis.

We also performed calculations of magnetically noncollinear spin spirals along the [111] axis using the FLAPW method. ${ }^{30}$ However, the results of Ref. 17 were not reproduced. Our results are shown in Fig. 12. Calculations within both the LSDA and the GGA show a ferromagnetic ground state (i.e., a global minimum at $\vec{q}=0$ ), while a local minimum is found close to $\vec{q}=(2 \pi / a)(0.45,0.45,0.45)$, i.e., at the point where the spin-spiral minimum is found in Ref. 17. The energy difference between the two (local and global) minima is of the order of $11 \mathrm{meV}$ within the LSDA and $13 \mathrm{meV}$ within the GGA. We do not know the origin of the discrepancy between our calculations and the calculations of Ref. 17. A possible source of discrepancy is the use of a spherical potential approximation in Ref. 17, as opposed to a full potential calculation here.

However, these noncollinear calculations (ours as well as the ones of Ref. 17) neglect a $12 \%$ Mn-Fe swap which is seen experimentally in $\mathrm{Fe}_{2} \mathrm{MnSi}$; i.e., experimentally this alloy has a small degree of disorder. Given the small calculated energy differences between the local and global minima (about $10 \mathrm{meV}$ per formula unit), a correct description of this swap can have important consequences. We attempted to model the swap by calculating the electronic structure of $\left(\mathrm{Fe}_{0.94} \mathrm{Mn}_{0.06}\right)_{\mathrm{A}, \mathrm{C}}\left(\mathrm{Fe}_{0.12} \mathrm{Mn}_{0.88}\right)_{\mathrm{B}} \mathrm{Si}$ within the CPA; here, the indexes $(\mathrm{A}, \mathrm{B}, \mathrm{C})$ refer to the corresponding positions in the unit cell. The magnetic structure was subsequently investigated in two ways. First, by calculating the exchange constants and relaxing the magnetic configuration at $T=0 \mathrm{~K}$ by a Monte Carlo simulation. Second, by calculating a disordered

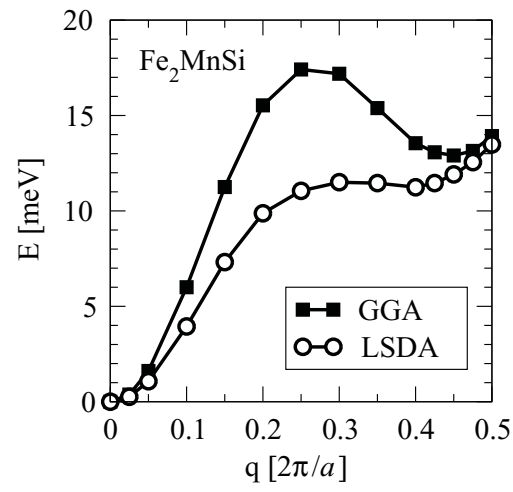

FIG. 12. Calculated flat spin-spiral dispersion relations of $\mathrm{Fe}_{2} \mathrm{MnSi}$ along the [111] direction within the LSDA and GGA. The abscissa $q$ corresponds to the point $(q, q, q) 2 \pi / a$ of the Brillouin zone. The FLAPW method was used in this calculation. 
local moment state at the $B$ site within the CPA (in the way described in Sec. IV). However, in all cases the outcome was a ferromagnetic ground state.

What is missing within the CPA description is the shortrange configurational order, i.e., the possibility to describe clustering of $\mathrm{Mn}_{\mathrm{A}, \mathrm{C}}$ atoms around $\mathrm{Mn}_{\mathrm{B}}$ atoms. We speculate that this swap and clustering causes the canting of some $\mathrm{Mn}_{\mathrm{B}}$ moments by interaction with the neighboring $\mathrm{Mn}_{\mathrm{A}, \mathrm{C}}$ atoms and is therefore essential for the appearance of the re-entrant behavior (such a scenario was already suggested in Ref. 9). Possibly, as the temperature is increased, the canting of the $\mathrm{Mn}_{\mathrm{B}}$ moments is reduced on the average and the total magnetization increases; this hypothesis would require a weak coupling of the canted $\mathrm{Mn}_{\mathrm{B}}$ moments to their $\mathrm{Mn}_{\mathrm{A}, \mathrm{C}}$ neighbors compared to the coupling of the noncanted $\mathrm{Mn}_{\mathrm{B}}$ moments to their $\mathrm{Fe}_{\mathrm{A}, \mathrm{C}}$ neighbors.

Our hypothesis is supported by the following experimental findings. (i) The re-entrant behavior and the swap appear in the same concentration range $(x>0.75)$. (ii) The re-entrant behavior is sensitive to the heat treatment of the alloy; after proper annealing, it was found that the re-entrant behavior smoothens, although $T_{\mathrm{R}}$ does not change. ${ }^{2}$ This, in conjunction with the calculations showing that the unswapped state is the ground state, suggests that annealing causes a fraction of the $\mathrm{Mn}$ atoms to return from the $A, C$ site to the $B$ site, so that the number of Mn clusters (and canted Mn moments, as we suspect) lessens. If this hypothesis is true, $T_{\mathrm{R}}$ should be indeed unaffected by annealing, because it would correspond to a finite-size effect (characteristic exchange-energy scale of a small cluster) rather than a phase transition. Such a possibility has been suggested by Nielsen and collaborators, ${ }^{41}$ based on Monte Carlo calculations of model systems. (iii) Clustering would result in a local environment which is closer to $\mathrm{Mn}_{3} \mathrm{Si}$, which is known to show antiferromagnetic behavior.

Furthermore, it is observed that the "smoothening" of the re-entrant behavior after annealing is accompanied by an increase of both $M_{0}$ and $M_{\text {extr }}$ (shown in Fig. 7 of Ref. 2 for $\mathrm{Fe}_{1.75} \mathrm{Mn}_{1.25} \mathrm{Si}$ ). We therefore make the plausible assumption that this behavior is present also at concentrations $x<0.75<1$, and that the ground state, with all Mn atoms being at the $B$ site, will show a higher magnetic moment than $M_{\text {extr }}$. This could resolve the discrepancy between the measured $^{2}$ magnetization value of $M_{\text {extr }} \approx 2.3 \mu_{\mathrm{B}}$ compared to calculated value of $3 \mu_{\mathrm{B}}$ in $\mathrm{Fe}_{2} \mathrm{MnSi}$.

First-principles investigations of such short-range-order effects require an approach beyond the CPA, e.g., by use of the nonlocal $\mathrm{CPA}^{42}$ or large-supercell techniques, ${ }^{43}$ and we therefore defer this study to a future work.

\section{CONCLUSION}

The electronic and magnetic structure of the magnetic intermetallic alloy $\mathrm{Fe}_{3-x} \mathrm{Mn}_{x} \mathrm{Si}$, for $0 \leqslant x \leqslant 1$ has been investigated using density-functional theory within the GGA, together with the CPA to describe disorder. We find that important experimental findings, such as the trends of the magnetization and of the Curie temperature as a function of concentration, are reproduced. They can be interpreted in terms of single-particle energies with the help of the density of states, by using simple physical arguments, namely symmetrydependent hybridization of wave functions and local charge neutrality. Quantitatively, the drop of the magnetization as a function of $\mathrm{Mn}$ concentration is underestimated, especially at $x>0.75$, however this could be due to Mn-Fe swap and Mn clustering in experiment that cannot be captured by the CPA; the same applies to the re-entrant behavior. It is therefore worthwhile to investigate $\mathrm{Fe}_{3-x} \mathrm{Mn}_{x} \mathrm{Si}$ beyond this approximation in the future.

However, it is rather surprising that the two most common approximations to DFT, namely the LSDA and the GGA, give ground states that differ at low concentration not only quantitatively but also qualitatively, although they almost agree on the single-particle spectrum. The superiority of GGA that we find here is common but certainly not general, which shows the need for theory to go hand in hand with experiment for the understanding of magnetic intermetallic compounds.

Concerning the relevance to spintronics, the most important finding here is the continuous variation of the spin polarization at $E_{\mathrm{F}}$ over a wide range, in the range $-0.3<P<1$ for $0<$ $x<0.75$, i.e., in the region where theory and experiment are in reasonable agreement and before the onset of the re-entrant behavior. Since the polarization at $E_{\mathrm{F}}$ is a property that is notoriously difficult to measure with precision, the variation which is found theoretically could be used to improve or calibrate the methods of measurement of $P$ in a single type of material, so that spurious effects in measurement can be treated on the same footing and understood better.

\section{ACKNOWLEDGMENTS}

We would like to thank the Supercomputing Center of the Forschungszentrum Jülich (JSC) for providing computational time. This work was funded in part by the Young Investigators Group Programme of the Helmholtz Association "Computational Nanoferronics Laboratory,” Contract No. VH-NG-409.

\footnotetext{
*m.lezaic@fz-juelich.de

†ph.mavropoulos@fz-juelich.de

${ }^{1}$ V. Niculescu, T. J. Burch, and J. I. Budnick, J. Magn. Magn. Mater. 39, 223 (1983).

${ }^{2}$ S. Yoon and J. G. Booth, J. Phys. F 7, 1079 (1977).

${ }^{3}$ S. Fujii, S. Ishida, and S. Asano, J. Phys. Soc. Jpn. 64, 185 (1995).

${ }^{4}$ K. Hamaya, H. Itoh, O. Nakatsuka, K. Ueda, K. Yamamoto, M. Itakura, T. Taniyama, T. Ono, and M. Miyao, Phys. Rev. Lett. 102, 137204 (2009).
}

${ }^{5}$ T. J. Burch, T. Litrenta, and J. I. Budnick, Phys. Rev. Lett. 33, 421 (1974).

${ }^{6} \mathrm{~S}$. Pickart, T. Litrenta, T. Burch, and J. I. Budnick, Phys. Lett. 53 A, 321 (1975).

${ }^{7}$ A. C. Swintendick, Solid State Commun. 19, 511 (1976).

${ }^{8}$ S. Yoon and J. G. Booth, Phys. Lett. 48 A, 381 (1974).

${ }^{9}$ V. Niculescu, K. Raj, T. J. Burch, and J. I. Budnick, Phys. Rev. B 13, 3167 (1976).

${ }^{10}$ K. R. A. Ziebeck and P. J. Webster, Philos. Mag. 34, 973 (1976). 
${ }^{11}$ S. H. Mahmood, A.-F. Lehlooh, A. S. Saleh, and F. E. Wagner, Phys. Status Solidi B 241, 1186 (2004).

${ }^{12}$ J. G. Booth, J. E. Clark, J. D. Ellis, P. J. Webster, and S. Yoon, Proceedings of the International Conference on Magnetism, Vol. IV, Nauka, Moscow (1974), p. 557.

${ }^{13}$ T. Nagano, S. Uwanuyu, and M. Kawakami, J. Magn. Magn. Mater. 140, 123 (1995).

${ }^{14}$ T. Ersez, S. J. Kennedy, and T. J. Hicks, J. Phys.: Condens. Matter 8, 7771 (1996).

${ }^{15}$ T. F. Smith, G. K. Bristow, C. V. Boughton, and P. R. W. Hudson, J. Phys. F 10, 2867 (1980).

${ }^{16}$ J. R. Miles, T. F. Smith, and T. R. Finlayson, Aust. J. Phys. 41, 781 (1988).

${ }^{17}$ P. Mohn and E. Supanetz, Philos. Mag. B 78, 629 (1998).

${ }^{18}$ A. Go, M. Pugaczowa-Michalska, and L. Dobrzyński, Eur. Phys. J. B 59, 1 (2007).

${ }^{19}$ B. Hamad, J. Khalifeh, I. A. Aljarayesh, C. Demangeat, H.-B. Luo, and Q.-M. Hu, J. Appl. Phys. 107, 093911 (2010).

${ }^{20}$ M. Szymański, M. Jankowski, L. Dobrzyński, A. Wiśniewski, and S. Bednarski, J. Phys.: Condens. Matter 3, 4005 (1991).

${ }^{21}$ P. J. Brown, K. R. A. Ziebeck, and J. M. Huntley, J. Magn. Magn. Mater. 50, 169 (1985).

${ }^{22}$ J. P. Perdew, J. A. Chevary, S. H. Vosko, K. A. Jackson, M. R. Pederson, D. J. Singh, and C. Fiolhais, Phys. Rev. B 46, 6671 (1992).

${ }^{23}$ S. H. Vosko, L. Wilk, and N. Nusair, Can. J. Phys. 58, 1200 (1980).

${ }^{24}$ M. Asato, A. Settels, T. Hoshino, T. Asada, S. Blügel, R. Zeller, and P. H. Dederichs, Phys. Rev. B 60, 5202 (1999).

${ }^{25}$ The SPR-TB-KKR package, H. Ebert and R. Zeller, [http://olymp.cup.uni-muenchen.de/ak/ebert/SPR-TB-KKR].

${ }^{26}$ N. Stefanou and R. Zeller, J. Phys.: Condens. Matter 3, 7599 (1991); N. Stefanou, H. Akai, and R. Zeller, Comput. Phys. Commun. 60, 231 (1990).

${ }^{27}$ M. Weinert, E. Wimmer, and A. J. Freeman, Phys. Rev. B 26, 4571 (1982).
${ }^{28} \mathrm{Ph}$. Kurz, F. Förster, L. Nordström, G. Bihlmayer, and S. Blügel, Phys. Rev. B 69, 024415 (2004).

${ }^{29}$ M. Ležaić, Ph. Mavropoulos, G. Bihlmayer, and S. Blügel (unpublished).

${ }^{30}[\mathrm{http}: / /$ www.flapw.de].

${ }^{31}$ A. I. Liechtenstein, M. I. Katsnelson, V. P. Antropov, and V. A. Gubanov, J. Magn. Magn. Mater. 67, 65 (1987).

${ }^{32}$ E. Sasioglu, L. M. Sandratskii, P. Bruno, and I. Galanakis Phys. Rev. B 72, 184415 (2005).

${ }^{33}$ I. Turek, J. Kudrnovský, V. Drchal, and P. Bruno, Philos. Mag. 86, 1713 (2006).

${ }^{34}$ M. Ležaić, Ph. Mavropoulos, and S. Blügel, Appl. Phys. Lett. 90, 082504 (2007).

${ }^{35}$ M. Pajda, J. Kudrnovský, I. Turek, V. Drchal, and P. Bruno, Phys. Rev. B 64, 174402 (2001).

${ }^{36}$ P. Buczek, A. Ernst, P. Bruno, and L. M. Sandratskii, Phys. Rev. Lett. 102, 247206 (2009).

${ }^{37}$ F. Lechermann, F. Welsch, C. Elsässer, C. Ederer, M. Fähnle, J. M. Sanchez, and B. Meyer, Phys. Rev. B 65, 132104 (2002).

${ }^{38}$ I. Galanakis, P. H. Dederichs, and N. Papanikolaou, Phys. Rev. B 66, 174429 (2002); I. Galanakis, Ph. Mavropoulos and P. H. Dederichs, J. Phys. D 39, 765 (2006).

${ }^{39}$ H. Kepa, T. J. Hicks, and R. L. Davis, Mater. Sci. Forum 27-28, 267 (1988).

${ }^{40}$ T. Ersez, S. J. Kennedy, and T. J. Hicks, Condens. Matter Phys. 7, 8423 (1995).

${ }^{41}$ M. Nielsen, D. H. Ryan, H. Guo, and M. Zuckermann, Phys. Rev. B 53, 343 (1996).

${ }^{42}$ M. Jarrell and H. R. Krishnamurthy, Phys. Rev. B 63, 125102 (2001); D. A. Rowlands, J. B. Staunton, and B. L. Györffy ibid. 67, 115109 (2003); D. Ködderitzsch, H. Ebert, D. A. Rowlands, and A. Ernst, New J. Phys. 9, 81 (2007).

${ }^{43}$ R. Zeller, J. Phys.: Condens. Matter 20, 294215 (2008). 